\title{
It's time to ramp up genetic counseling training
}

\author{
Myra I. Roche, MS, CGC ${ }^{1}$ and Anne E. Greb, MS, CGC ${ }^{2}$
}

Amid the rumors of hefty signing bonuses, coverage of transcontinental moving expenses, and, yes, even full repayment of educational loans, it may strain credibility to believe that this frenetic economic wooing is directed at...genetic counselors? Indeed, the employment opportunities in the field have never been more robust. One recent estimate cited 1,500 available jobs for the approximately 4,000 board-certified genetic counselors, most of whom, by the way, already have jobs. After years of promoting their value and persistently redefining their menu of expanded roles while simultaneously preserving a unique core skill set, genetic counselors have doggedly pursued this moment. As a consequence, the "expand the workforce" drumbeat, a sound we have heard many times before, has taken on the intensity of a brood of 17 -year cicadas hatching on an early Carolina summer evening.

In many people's minds, the question about workforce expansion is no longer "if"; it has finally become "how." The current flood of opportunities begs to be exploited by flinging open every possible spigot in the pipeline. Increased awareness of the profession at both high school and college levels has modestly swelled the applicant pool size to approximately 800 per year. But this comparative trickle of incoming flow is quickly absorbed by limited training program capacities further dampening already low acceptance rates.

Which obstructive factors account for the logjam, and can they be disentangled to pry open the floodgates? In their article "Expanding the Genetic Counseling Workforce: Program Directors' Views on Increasing the Size of Genetic Counseling Graduate Programs," Pan and coauthors ${ }^{1}$ asked program directors to name the critical forces controlling the inelasticity in genetic counseling training. The data obtained from surveys and from interviews of 25 of the 34 program directors identified multiple interrelated barriers to expansion. Yet, barriers aside, $73 \%$ of directors agreed that the current training rate is too slow.

On the surface, the math seems simple enough: increase the number of programs, increase the typical class sizes, or both. The number of programs has, in fact, more than tripled in the past 30 years; however, three times a small number $(\sim 10)$ is still a small number $(\sim 30)$. New applications for training programs continue to be submitted, but most are not located in the large urban areas that are most capable of supporting the number of rotation sites needed to admit larger class sizes.
Even though program directors expressed a willingness to expand class sizes, using the current educational training models it is unrealistic for them to, for example, triple their number of students. At current rates, Pan et al. estimated that fewer than 2,400 genetic counselors would be added over the next 10 years. Even after factoring in our sluggish 5\% growth rate, that number would barely exceed 3,100. By comparison, the $17 \%$ growth rate of physician assistants seems almost oncogenic. And 17\% of a big number is a really big number; in 2013, there were more than 95,000 certified physician assistants. ${ }^{2}$ Although this magnitude of growth may be as undesirable as it is currently unattainable, the eagerness to expand the number of genetic counselors has been, well, growing.

In its current form, the clinical rotation exemplifies both the tremendous power of traditional individualized training and the tremendous restrictions it places on the number of students who can be trained. Program directors described the matching of students to rotations as an ever-changing series of interlocking parts that are under constant threat of being swamped by everyday life events; a supervisor's unexpected maternity leave, adverse weather that prevent students' travel to sites, and institutional policies that delay their access to clinics. This precariousness precludes programs from accepting the maximum number of students, further compromising expansion efforts. That this patchwork system even manages to stay afloat can be attributed to a small cadre of professionals willing to guide individual students through the labyrinthine passageway to achieving clinical competence. But economic forces are incessantly eroding the reservoir of clinical mentors and, by extension, their largely gratis mentoring activities. Their continued attrition, via more lucrative job offers, and, for those who remain behind, increasing workloads that make mentoring unimaginable threaten to further constrict the training capabilities of clinical sites.

By objective measures, such as high certification examination passing rates, the genetic counseling training model has shown success at producing competent genetic counselors. At the heart of this model are the personalized student-mentor relationships that have been characteristic since the earliest days of genetic counseling training. Although clearly beneficial to students and gratifying to their instructors, these methods have been resistant to even modest scale-up efforts. In addition, academic environments, where much of the training, both didactic and

${ }^{1}$ Departments of Pediatrics and Genetics, University of North Carolina at Chapel Hill, Chapel Hill, North Carolina, USA; ${ }^{2}$ Joan H. Marks Graduate Program in Human Genetics, Sarah Lawrence College, Bronxville, New York, USA. Correspondence: Myra I. Roche (myra_roche@med.unc.edu)

Submitted 20 January 2016; accepted 25 January 2016; advance online publication 7 April 2016. doi:10.1038/gim.2016.25 
clinical, occurs, no longer represent the quintessential working environment for genetic counselors. The traditional training model, born and raised in an academic environment, may not be capable of the flexibility needed to significantly train more students for increasingly diverse roles.

Experimentation with different components of the training model could reveal just how far the envelope of individual mentoring can be pushed without unduly sacrificing the end result. Although often perceived as an ideal, a one-to-one training ratio may actually not be optimal for some students in some environments. Rotation schedules that allow students to become immersed by full-time attendance for fewer weeks could replace typical part-time, longer rotations that could potentially benefit both students and supervisors. Block schedules, in which weeks of time are devoted to either rotations or classroom, have been successfully implemented in other educational settings such as medical schools and could facilitate an increased class size. Virtual training of clinical skills could enhance students' preparedness for rotations, and a broader use of technology could increase their access to a wider variety of experiences. Finally, logbook requirements could be revised to define and recognize how a wider variety of learning environments can successfully contribute to the development of the core set of genetic counselor skills.

There is an understandable hesitancy against tinkering with a complex system that has worked well in the past and is not flagrantly broken. But a widening gap between the number of available jobs and the number of available genetic counselors may embolden other professionals to colonize many of our hard-earned expanded niches. It remains to be seen whether or not the current hot job market is signaling a long-term trend or if it represents a moment in time that will largely dissipate as sought-after skills, such as variant annotation, become more automated. But academic training programs that are notoriously burdened with Queen Mary-like turning ratios, making even modest changes difficult to implement, cannot afford the luxury of waiting until the signals are crystal clear. Based on past technological advances that have led to dramatic increases in the population who are eligible for genetic services, it should be evident that even our most vigorous attempts to increase the supply of genetic counselors are unlikely to match, let alone exceed, their employment opportunities, at least in the foreseeable future.

Thirty years ago, it was axiomatic that most genetic counselors would be employed in academic medical centers performing roles that eerily matched those of their clinical supervisors. And while the successful expansion of these roles is directly related to the current robust employment outlook, this diversity also makes it difficult to anticipate which critical combination of educational and clinical experiences can successfully launch a fledgling genetic counselor into the next decade. Reimagining the way we train genetic counselors will also force us to grapple with redefining our essence. But we should be used to that by now.

\section{ACKNOWLEDGMENTS}

The authors gratefully acknowledge Catherine Reiser, Bonnie LeRoy, and Randi Stewart for sharing their perspectives.

\section{DISCLOSURE}

The authors declare no conflict of interest.

\section{REFERENCES}

1. Pan V, Yashar BM, Pothast R, Wicklund C. Expanding the genetic counseling workforce: program directors' views on increasing the size of genetic counseling graduate programs. Genet Med; e-pub ahead of print 7 January 2016.

2. NCCPA 2013 Statistical Profile of Certified Physician Assistants. An Annual Report of the National Commission on Certification of Physician Assistants. 2014. https://www.nccpa.net/Upload/PDFs/2013StatisticalProfileofCertifiedPhy sicianAssistants-AnAnnualReportoftheNCCPA.pdf. Accessed 19 January, 2016. 